\title{
Lipid Bilayer-Enabled Synthesis of Waxberry-like Core-Fluidic Satellite Nanoparticles: Toward Ultrasensitive Surface-Enhanced Raman Scattering Tags for Bioimaging
}

\author{
Rongchao Mei, ${ }^{\dagger+}$ Yunqing Wang, ${ }^{* \dagger,}$ Wanhui Liu, ${ }^{\dagger}$ and Lingxin Chen ${ }^{*}, \dagger, \S_{\odot}$
}

${ }^{\dagger}$ CAS Key Laboratory of Coastal Environmental Processes and Ecological Remediation, Research Center for Coastal Environment Engineering Technology of Shandong Province, Yantai Institute of Coastal Zone Research, Chinese Academy of Sciences, Yantai 264003, China

${ }^{\ddagger}$ School of Pharmacy, Collaborative Innovation Center of Advanced Drug Delivery System and Biotech Drugs in Universities of Shandong, Key Laboratory of Molecular Pharmacology and Drug Evaluation (Yantai University), Ministry of Education, and ${ }^{\S}$ College of Chemistry and Chemical Engineering, Yantai University, Yantai 264005, China

Supporting Information

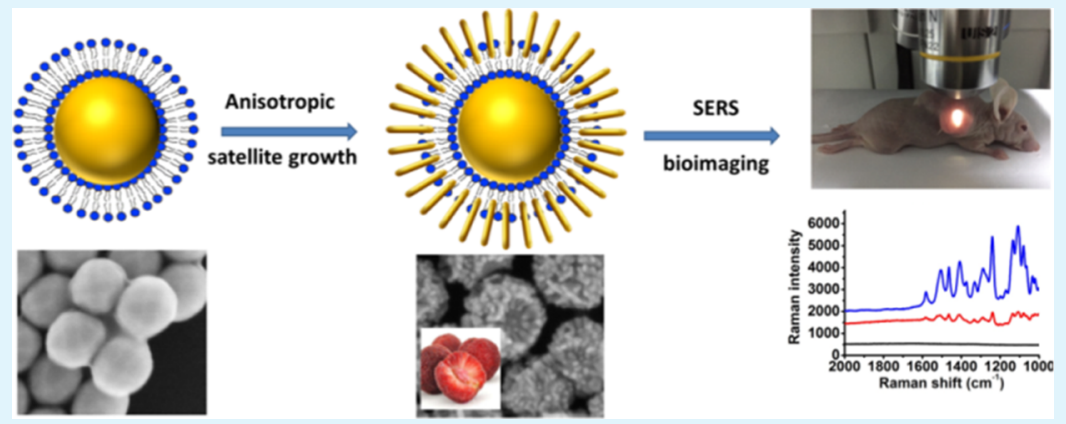

ABSTRACT: Herein, we presented waxberry-like core-satellite (C-S) nanoparticles (NPs) prepared by an in situ growth of satellite gold NPs on spherical phospholipid bilayer-coated gold cores. The fluidic lipid bilayer cross-linker was reported for the first time, which imparted several novel morphological and optical properties to the $\mathrm{C}-\mathrm{S}$ NPs. First, it regulated the anisotropic growth of the satellite NPs into vertically oriented nanorods on the core NP surface. Thus, an interesting waxberry-like nanostructure could be obtained, which was different from the conventional raspberry-like C-S structures decorated with spherical satellite NPs. Second, the satellite NPs were "soft-landed" on the lipid bilayer and could move on the core NP surface under certain conditions. The movement induced tunable plasmonic features in the C-S NPs. Furthermore, the fluidic lipid bilayer was capable of not only holding an abundance of reporter molecules but also delivering them to the hotspots at the junctions between the core and satellite NPs, which made the C-S NPs an excellent candidate for preparing ultrasensitive surface-enhanced Raman scattering (SERS) tags. The bioimaging capabilities of the C-S NP-based SERS tags were successfully demonstrated in living cells and mice. The developed SERS tags hold great potential for bioanalysis and medical diagnostics.

KEYWORDS: gold nanoparticle, core-satellite nanoparticle, in situ growth, lipid bilayer, surface-enhanced Raman scattering tags, bioimaging

\section{INTRODUCTION}

Surface-enhanced Raman scattering (SERS) tags, which are composed of noble metal nanoparticles (NPs) and Raman reporters, have demonstrated great potential in applications such as biochemical analysis owing to their sensitivity and multiplex labeling ability. ${ }^{1-5}$ The structure of noble metal NPs plays a crucial role in the optical features of SERS tags. ${ }^{6-9}$ In recent years, plasmonic core-satellite $(C-S)$ nanostructures have drawn much attention as novel SERS substrates for the preparation of SERS tags. Numerous built-in isotropic hotspots create the strong electromagnetic enhancement ability between the cores and the satellites, therefore rendering the SERS tags with excellent sensitivity. ${ }^{3,10-14}$
The predominant approach to fabricate such $\mathrm{C}-\mathrm{S}$ nanostructures was a bottom-up strategy using as-synthesized NPs [usually gold nanospheres (NSs)] as the cores and smaller gold or silver NPs as the satellites. ${ }^{15-18}$ Organic linker molecules are usually applied to create a permanent link between the core and satellite NPs. ${ }^{3}$ The main types of linkers are (1) small thiol group-functionalized molecular linkers, such as cysteamine, ${ }^{15}$ (3-mercaptopropyl)trimethoxysilane, ${ }^{16}$ amino-functionalized heterobifunctional Raman reporter, ${ }^{12}$ and $p$-aminothiophenol ${ }^{17,19,20}$ and (2), macromolecular

Received: April 17, 2018

Accepted: June 25, 2018

Published: June 25, 2018 
compounds (mercapto polyethylene glycol, ${ }^{21}$ DNA pairs, ${ }^{10,22,23}$ proteins, ${ }^{24,25}$ etc.). These methods could yield well-defined nanocluster systems with a precise structural order, relative geometry, and interparticle distance. ${ }^{24}$ Another approach was to modify the Au cores with single-stranded DNA, $^{26}$ poly-L-histidine, ${ }^{27,28}$ or a specific small molecule ${ }^{11}$ with a strong affinity for noble metal ions, followed by an in situ synthesis of satellite NPs on the cores. This method was advantageous from the perspective of scalability because the yield of the resulting $\mathrm{C}-\mathrm{S} \mathrm{NP}$ products was high and the separation process of unbound satellites could be avoided. However, the morphology of the in situ overgrowth product was determined by the modified linker molecules on the gold core. Under normal conditions, only continuous metal shells formed on the core. ${ }^{11}$ So far, only a few linkers have been reported that can precisely control the growth of surface NPs into discontinuous satellites, eventually forming the $\mathrm{C}-\mathrm{S}$ nanostructures.

A phospholipid bilayer is a promising Au NP linking material, taking advantage of the $\mathrm{Au}-\mathrm{N}$ interaction between the Au NP and quaternary ammonium headgroup of the lipid molecules. $^{29-31}$ Until now, two predominant types of lipid bilayer-Au hybrid structures have been reported. One structure consists of the Au NP cores encapsulated in lipid bilayers. ${ }^{32-37}$ In this hybrid structure, the lipid bilayer coating improved the biocompatibility, colloidal stability, targeting capability, and versatility of the Au cores for the preparation of multiplex labeling of SERS tags. ${ }^{8,38,39}$ The other structure consisted of liposome surfaces decorated with small $\mathrm{Au}$ clusters. In this structure, the liposomes served as flexible scaffolds for controlling the self-assembly of small Au clusters into densely packed shells, ${ }^{40}$ which maintained similar optical characteristics [such as near-infrared (NIR) light absorption] as those of solid metallic shells. However, the Au-decorated liposome could biodegrade into small Au clusters in detergentrich environments or under photothermal treatment. This unique feature made it suitable to be served as a lightresponsive nanomedicine. ${ }^{41-46}$ Moreover, a novel liposomebased SERS substrate was also reported, in which the Raman reporter was encapsulated inside the aqueous compartment of the liposome, preventing the reporter from directly coming into contact with the metallic surfaces. ${ }^{47}$

In this work, we tried to integrate the above two structures and produce novel $\mathrm{C}-\mathrm{S}$ NPs by using lipid bilayers as crosslinkers, which imparted several attractive properties to the C$S$ NPs. First, the lipid bilayer regulated the anisotropy in the satellite NPs, growing into a nanorod array and orienting vertically on the surface of the NP core owing to its fluidic feature. Thus, interestingly, waxberry-like C-S NPs, instead of conventional raspberry-like structures (with spherical satellites), could be obtained. Second, the satellite NPs were "softlanded" on the lipid bilayer and were movable on the core NP surface, which would induce tunable absorption and SERS features of the $\mathrm{C}-\mathrm{S}$ NPs. Furthermore, the C-S NPs exhibited an extraordinary SERS enhancement ability owing to a large number of hotspots at the $\mathrm{C}-\mathrm{S}$ and satellite-satellite junctions, which were separated by the fluidic lipid bilayers. Various kinds of Raman reporters could be readily entrapped in the lipid bilayers and be dispersed to these hotspots, reaching the maximum SERS signal enhancement. On the basis of their ultrasensitivity, SERS tags were further fabricated, and the bioimaging capabilities were successfully demonstrated in living cells and mice.

\section{EXPERIMENTAL SECTION}

Materials. Cetyltrimethylammonium bromide (CTAB), cetyltrimethylammonium chlorine (CTAC), 3,3'-diethylthiatricarbocyanine iodide (DTTC), 3,3'-diethylthiadicarbocyanine iodine (DTDC), 4nitrothiophenol (4-NT), and dopamine were obtained from SigmaAldrich. Chloroauric acid $\left(\mathrm{HAuCl}_{4}\right)$, sodium borohydride $\left(\mathrm{NaBH}_{4}\right)$, L-ascorbic acid (AA), crystal violet $(\mathrm{CV})$, sodium bromide $(\mathrm{NaBr})$, ethanol, malachite green (MG), Nile blue (NB), coomassie brilliant blue (CBB), rhodamine 6G (R6G), and Triton X-100 were purchased from Sinopharm Chemical Reagent Co. Ltd. MG isothiocyanate (MGITC) was obtained from Life technologies. DIR $\left(1,1^{\prime}\right.$ Dioctadecyl-3,3,3' $3^{\prime}$-tetramethylindotricarbocyanine) was purchased from AAT Bioquest. HEPES (4-(2-Hydroxyethyl)-1-piperazineethanesulfonic acid) was obtained from Life Science Products. Cyanine 7 (Cy7) monoacid was synthesized in our laboratory. Lipoid E80 was purchased from Lipoid GmbH. According to the manufacturer's specifications, it was composed of $80 \%$ phosphatidylcholine, $8 \%$ phosphatidylethanolamine, $3.6 \%$ nonpolar lipids, and $2 \%$ sphingomyelin. Dipalmitoyl-sn-glycero-3-phosphocholine (DPPC) was obtained from Corden Pharma. Dulbecco's modified Eagle's medium (DMEM), fetal bovine serum, penicillin-streptomycin solution, and trypsin-ethylenediaminetetraacetic acid solution were purchased from Thermo Scientific. Deionized water was used in all the experiments.

Characterization. The UV/vis/NIR absorption spectra were recorded on a Thermo Scientific NanoDrop 2000/2000C spectrophotometer. The SERS spectra were recorded by using a DXR Raman microscope (Thermo Scientific, USA). A 632.8 or $780 \mathrm{~nm}$ laser was focused by a $10 \times$ and a $50 \times$ microscope objective for the sample solution, cell or mouse measurement, respectively. The zeta potential and dynamic light scattering (DLS) size were measured on a Zetasizer NanoZS90 (Malvern Instruments, Malvern, U.K.). Transmission electron microscopy (TEM) images were acquired on a JEM-1400 transmission electron microscope (JEOL, Japan). Scanning electron microscopy (SEM) images were obtained on an S-4800 field emission scanning electron microscope (Hitachi, Japan).

Synthesis of Au NSs. Au NSs that served as core were synthesized according to a reported method with some minor modifications. ${ }^{48}$ Briefly, the seed solution was prepared by reducing $\mathrm{HAuCl}_{4}(0.5 \mathrm{mM}, 5 \mathrm{~mL})$ in CTAB $(0.2 \mathrm{M}, 5 \mathrm{~mL})$ with freshly prepared ice-cold $\mathrm{NaBH}_{4}(10 \mathrm{mM}, 0.6 \mathrm{~mL})$ at $27^{\circ} \mathrm{C}$. After $3 \mathrm{~h}, 50 \mu \mathrm{L}$ of the synthetic seed solution was mixed with CTAC $(0.2 \mathrm{M}, 2 \mathrm{~mL})$, AA $(0.1 \mathrm{M}, 1.5 \mathrm{~mL})$, followed by the addition of $\mathrm{HAuCl}_{4}$ solution $(0.5 \mathrm{mM}, 2 \mathrm{~mL})$. The reaction was allowed to continue at $27{ }^{\circ} \mathrm{C}$ for $15 \mathrm{~min}$. The product was collected by centrifugation twice at 14000 rpm for $40 \mathrm{~min}$ and then concentrated to $1 \mathrm{~mL}$. The diameter of the product was $10 \mathrm{~nm}$. Finally, CTAC $(0.1 \mathrm{M}, 40 \mathrm{~mL})$, AA (10 mM, 2.6 $\mathrm{mL})$, and the $10 \mathrm{~nm}$ seeds $(100 \mu \mathrm{L})$ were mixed, followed by the addition of $\mathrm{HAuCl}_{4}$ solution $(0.5 \mathrm{mM}, 40 \mathrm{~mL})$ using a syringe pump at an injection rate of $40 \mathrm{~mL} / \mathrm{h}$. The reaction was allowed to proceed at $27{ }^{\circ} \mathrm{C}$ for $10 \mathrm{~min}$ after the injection had been finished.

Synthesis of Liposomes. Liposomes were prepared by a conventional film hydration method. ${ }^{8}$ Briefly, the phospholipid was dissolved in ethanol $(5 \mathrm{mg} / \mathrm{mL})$, followed by the removal of the solvent using a rotary evaporator at $35^{\circ} \mathrm{C}$. A dried lipid film was obtained by further placing it under vacuum for $2 \mathrm{~h}$ at $40{ }^{\circ} \mathrm{C}$. The dried film was fully hydrated in $5 \mathrm{~mL}$ of water to form multilamellar vesicles, which were then broken into small unilamellar vesicles by using probe sonication $(150 \mathrm{~W}, 30 \mathrm{~min})$, indicated by the solution turning from turbid to clear.

Synthesis of Lipid Bilayer-Coated Au NSs. A volume of $1 \mathrm{~mL}$ of $\mathrm{Au}$ NS solution was centrifuged at $3000 \mathrm{rpm}$ for $10 \mathrm{~min}$, and the supernatant was discarded. The NS pellet was resuspended in $1 \mathrm{~mL}$ of liposome solution $(5 \mathrm{mg} / \mathrm{mL})$ and incubated at room temperature overnight. Then, the mixture was centrifuged at $3000 \mathrm{rpm}$ for $10 \mathrm{~min}$, and the pellet was dispersed in $50 \mu \mathrm{L}$ of water, followed by a dropwise addition of the dispersion into $1 \mathrm{~mL}$ of the liposome solution and stirring for $4 \mathrm{~h}$ at $50{ }^{\circ} \mathrm{C}$. The lipid bilayer-coated Au NSs were finally 


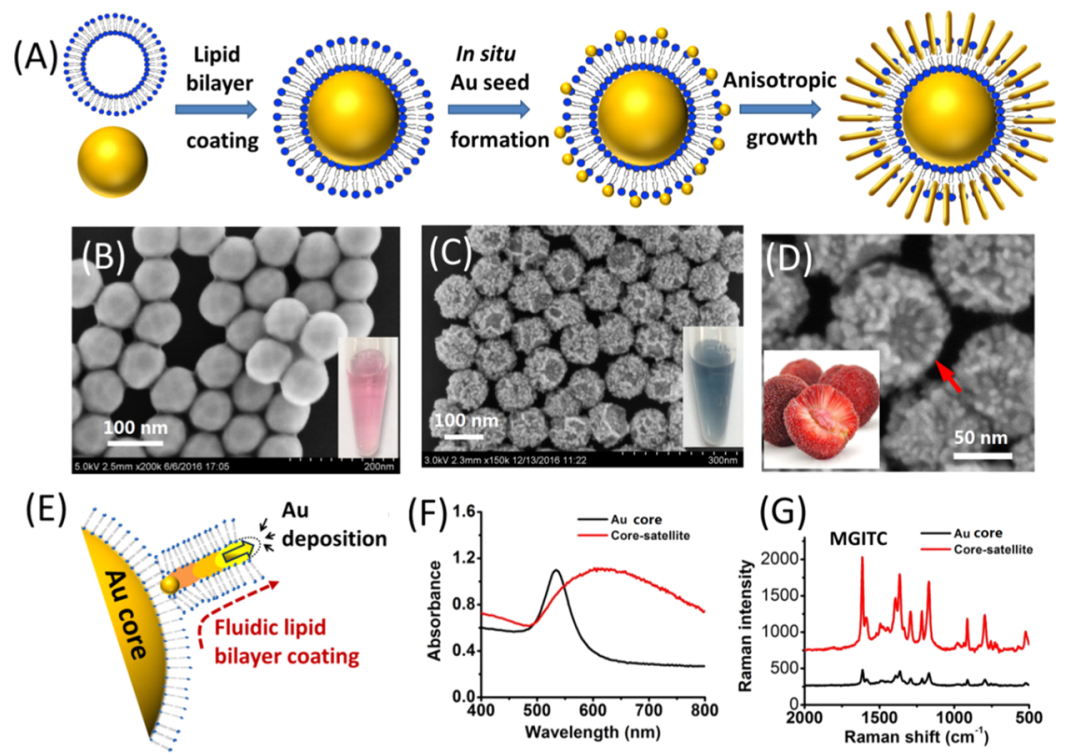

Figure 1. (A) Schematic illustration of lipid bilayer-assisted synthesis of waxberry-like C-S NPs. (B) SEM image and the solution color of $80 \mathrm{~nm}$ CTAC-stabilized Au NS cores. (C) SEM image and the solution color of C-S NPs prepared from the lipid bilayer-coated cores by the reaction with $2.5 \mathrm{mM} \mathrm{Au}^{3+}$ and $0.1 \mathrm{M} \mathrm{AA}$. (D) SEM image of the NPs with higher magnification illustrating a waxberry-like structure. The arrow indicates nanorod-shaped satellites. (E) Formation mechanism of nanorod-shaped satellite NPs. The fluidic lipid bilayer moved to and protected the side of the satellite NPs, and the Au atoms preferentially deposited on the "bare" tips. (F) UV-vis spectra of the Au core and C-S NPs. (G) SERS spectra of the MGITC Raman reporter obtained from the Au core and C-S NPs.

obtained by centrifuging the mixture at $4000 \mathrm{rpm}$ for $10 \mathrm{~min}$ and dispersing in $1 \mathrm{~mL}$ of water.

Synthesis of C-S NPs by $\mathrm{Au}^{3+}$. A volume of $1 \mathrm{~mL}$ of the bilayercoated Au NSs $\left(\mathrm{OD}_{540 \mathrm{~nm}}=1.0\right)$ was mixed with AA $(0.1 \mathrm{M}, 0.1 \mathrm{~mL})$, followed by the addition of $0.1 \mathrm{~mL}$ of $\mathrm{HAuCl}_{4}$ with different concentrations $(0.5,1,2.5$, and $5 \mathrm{mM})$ under vigorous stirring at room temperature.

Synthesis of $\mathrm{C}-\mathrm{S}$ NPs by $\mathrm{Au}^{+} . \mathrm{Au}^{+}$solution was first prepared according to a previous work. ${ }^{49}$ Briefly, $0.2 \mathrm{~g}$ of $\mathrm{NaBr}$ was dissolved in $3 \mathrm{~mL}$ of water followed by the addition of a different concentration of $\mathrm{HAuCl}_{4}(50 \mathrm{mM})$. AA $(0.5 \mathrm{M})$ solution was added dropwise to the mixture until its color turned from yellow to colorless, indicating the formation of $\mathrm{Au}^{+}$solution with varied concentrations (0.5, 1, 2.5, 5, and $10 \mathrm{mM})$. Then, $\mathrm{C}-\mathrm{S}$ NPs were synthesized by using $\mathrm{Au}^{+}$solution as the reaction material with the method similar to that using $\mathrm{Au}^{3+}$ solution.

Synthesis of Polydopamine-Coated C-S NPs. Polydopaminecoated C-S NPs were acquired according to a reported method. ${ }^{50}$ Briefly, $1 \mathrm{~mL}$ of C-S NPs was centrifuged at $2500 \mathrm{rpm}$ for $10 \mathrm{~min}$, and the precipitates were redispersed in $1 \mathrm{~mL}$ of $10 \mathrm{mM}$ Tris- $\mathrm{HCl}$ buffer ( $\mathrm{pH} 8.5$ ), followed by the addition of $30 \mu \mathrm{L}$ of $1 \mathrm{mg} / \mathrm{mL}$ dopamine hydrochloride solution under shaking. Then, the mixture was incubated at $37^{\circ} \mathrm{C}$ for $1 \mathrm{~h}$. The polydopamine-coated NPs were finally rinsed with water by centrifugation at $2600 \mathrm{rpm}$ for $10 \mathrm{~min}$.

Synthesis of Au Nanoflowers. Gold nanoflowers were synthesized according to a previous work. ${ }^{51}$ Briefly, $\mathrm{HAuCl}_{4}(20$ $\mathrm{mM}, 0.25 \mathrm{~mL}$ ) was added to $10 \mathrm{~mL}$ of HEPES solution ( $\mathrm{pH} 7.4,10$ $\mathrm{mM}$ ). The initial light yellow color of the mixture changed to blue in $30 \mathrm{~min}$, indicating the formation of Au nanoflowers.

Synthesis of C-S NP-Based SERS Tags. The SERS tags were obtained by mixing $500 \mu \mathrm{L}$ of C-S NPs and $15 \mu \mathrm{L}$ of different Raman reporters $\left(10^{-5} \mathrm{M}\right)$ for $0.5 \mathrm{~h}$. The Raman reporters were DTTC, DTDC, Cy7, DIR, CV, MGITC, MG, CBB, NB, and R6G. The NTlabeled SERS tags were obtained by mixing $500 \mu \mathrm{L}$ of C-S NPs and $150 \mu \mathrm{L}$ of NT $\left(10^{-4} \mathrm{M}\right)$ for $0.5 \mathrm{~h}$.

Cell Culture and Imaging. SMMC7721 cells (a liver cancer cell line) were grown as a monolayer in a humidified incubator at $37^{\circ} \mathrm{C}$ in air $/ \mathrm{CO}_{2}$ (95:5). The medium was DMEM that was supplemented with $10 \%$ fetal bovine serum. For all experiments, the cells were washed with phosphate-buffered saline (PBS) and harvested by using trypsin. All cells were finally resuspended in a fresh medium before plating. For cell imaging, the SMMC7721 cells $\left(2 \times 10^{4}\right)$ were seeded onto glass coverslips in a 24 -well plate with a $500 \mu \mathrm{L}$ of DMEM medium and cultured for $12 \mathrm{~h}$ to allow the cells to attach. Then, 150 $\mu \mathrm{L}$ of each SERS tag $(45 \mu \mathrm{g} / \mathrm{mL})$ was added into the cell culture. After incubation for $3 \mathrm{~h}$, the cell monolayer on the coverslip was repeatedly washed with PBS to remove the remaining particles and then sealed with a glass microscope slide. The observations were performed by Raman microscopy, and the SERS mapping could be performed at the same time.

In Vivo SERS Detection. The in vivo detection of the DTTClabeled NIR SERS tags was performed in two ways. One was to inject $200 \mu \mathrm{L}$ of the tags $(75 \mu \mathrm{g} / \mathrm{mL})$ directly into the tumor of a model of tumor-bearing mouse (xenograft tumor of A549 human lung cancer cells), followed by the detection of the SERS spectrum inside the tumor using Raman microscopy with a $780 \mathrm{~nm}$ laser $(150 \mathrm{~mW})$. The control spectrum was taken in an area away from the injected site. The other one was to inject $200 \mu \mathrm{L}$ of the tags $(150 \mu \mathrm{g} / \mathrm{mL})$ into a tumor-bearing mouse intravenously via the tail vein. The SERS spectra from the liver, the tail, and the tumor sites were recorded at different time intervals with $780 \mathrm{~nm}$ laser irradiation $(150 \mathrm{~mW})$. After $3.5 \mathrm{~h}$, the mouse was executed and dissected, and the signals from the tumor and the main organs were measured.

\section{RESULTS AND DISCUSSION}

Synthesis and Morphology of C-S NPs. The synthesis procedure of the C-S NPs is schematically illustrated in Figure 1A. The lipid bilayer-coated Au cores were prepared, followed by the addition of $\mathrm{Au}$ ions and AA for the in situ synthesis of satellite NPs. The Au seeds were first attached on the lipid bilayer and then anisotropically grew into rod-like satellite NPs.

Figure 1B shows the SEM images of the CTAC-stabilized gold NSs (core NPs) with a diameter of $80 \mathrm{~nm}$. After incubating and washing with liposome (DLS diameter: $79 \mathrm{~nm}$, zeta potential: $-18 \mathrm{mV}$ ) solutions, CTAC could be displaced by a phosphatidylcholine lipid that had a quaternary ammonium headgroup similar to the CTAC molecule, and 
(A)

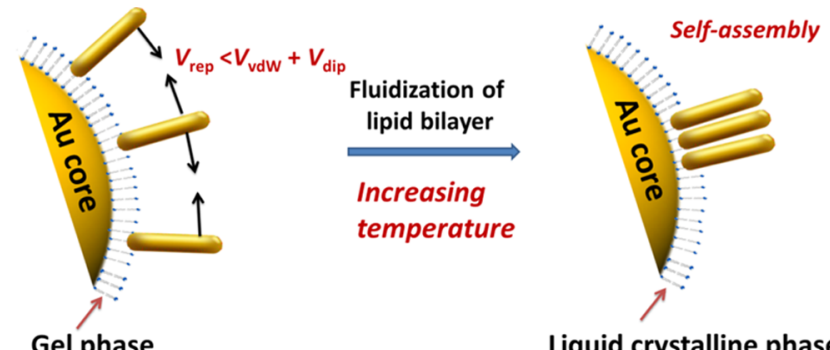

Gel phase

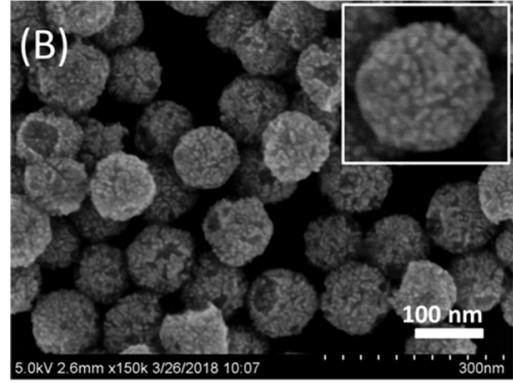

Liquid crystalline phase

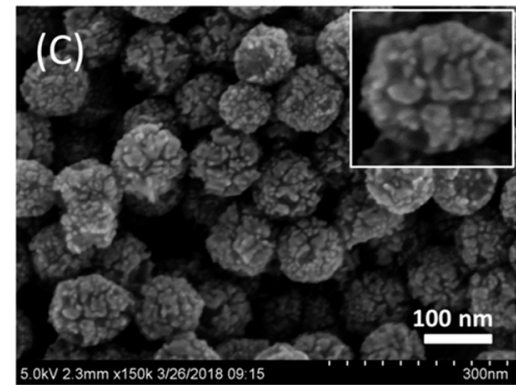

Figure 2. (A) Schematic illustration of heat-induced satellite aggregation on the surface of the Au core NPs. (B,C) NP morphologies before and after, respectively, in situ satellite aggregation on individual cores.

the lipid bilayer-coated Au core NPs could be obtained. The lipid layer could be verified by the zeta potential variation from $46.8 \mathrm{mV}$ of the CTAC-stabilized Au core to $-11.7 \mathrm{mV}$ of the lipid-coated products (Figure S1A). The TEM image of the lipid-coated $\mathrm{Au}$ cores shows the clear, diffuse coronas of the lipid bilayer (Figure S1B). The Fourier-transform infrared spectroscopy (FTIR) spectra of Lipoid E80, lipid-coated Au NS core, and CTAC-Au NS core were also recorded. As shown in Figure S1C, the characteristic peaks of phospholipids including carbonyl $\nu(\mathrm{C}=\mathrm{O})\left(1735 \mathrm{~cm}^{-1}\right)$, phosphate $\nu_{\mathrm{a}}\left(\mathrm{PO}_{2}^{-}\right)\left(1238 \mathrm{~cm}^{-1}\right)$, and $\nu_{\mathrm{s}}(\mathrm{C}-\mathrm{O}-\mathrm{C})\left(1091 \mathrm{~cm}^{-1}\right)$ could be clearly observed in the lipid-coated Au NS core. ${ }^{32,52}$ For the CTAC-NPs without liposome treatment, only weak peaks of alkyl chains appeared to be originating from the remaining CTAC ligand. The above results proved that the Au core was coated by lipid bilayers.

Furthermore, AA and chloroauric acid were added to the suspension of the lipid bilayer-coated Au cores in sequence. An abrupt color change occurred from the characteristic pink of the $\mathrm{Au}$ core to blue, indicating the formation of $\mathrm{C}-\mathrm{S}$ NPs (Figure 1B,C, insets). The SEM images (Figure 1C) revealed that the C-S NPs exhibited a branched morphology with a size of approximately $100 \mathrm{~nm}$. The DLS results (Figure S1B) also showed an increasing size of $105 \mathrm{~nm}$ compared to that of the Au NS core $(83 \mathrm{~nm})$ and a uniform size distribution with a polydispersity index of 0.15 . The image at higher magnification (Figure 1D) displayed that the satellite NPs were not conventional spheres but nanorods with an average width of $5 \mathrm{~nm}$ and a length of $25 \mathrm{~nm}$. The nanorods were perpendicular to the surface of the Au NS core and formed a layer of densely packed shells on the core, giving the $\mathrm{C}-\mathrm{S}$ NPs an interesting waxberry-like structure. The novel structure was clearly distinguished from the previously reported raspberry-like ones with spherical Au satellites.

We hypothesized that the fluidic property of the lipid bilayer was responsible for the formation of the unique, rod-shaped satellites. The transition temperature $\left(T_{\mathrm{m}}\right)$ of the lipid we used (Lipoid E80) was approximately $-8{ }^{\circ} \mathrm{C}$, and the bilayer was fluidic at the synthesis temperature $\left(25^{\circ} \mathrm{C}\right)$. As the seeds grow, the fluidic lipid bilayers tend to leave the Au core surface and be preferentially capped on the side of freshly generated $\mathrm{Au}$ satellite domains through strong $\mathrm{N}-\mathrm{Au}$ interactions. ${ }^{53}$ It was difficult to deposit $\mathrm{Au}$ in the lipid bilayer-protected regions, as the $\mathrm{Au}$ atoms preferred to be deposited on the tips where the capping ligands were relatively deficient, ${ }^{54,55}$ thus leading to the anisotropic growth of the seeds and the resultant nanorod array on the surface of the NP core (Figure 1E).

To further verify this mechanism, two control experiments were performed. First, blank Lipoid E80 liposomes served as a scaffold for fabricating a gold NP-liposome hybrid via the same AA reduction protocol. Similar rod-like NP arrays could also be observed on the hybrid NPs (Figure S2A). Second, the $\mathrm{Au}$ NS cores were coated by a DPPC lipid bilayer with the same headgroup as the Lipoid E80 lipid bilayer but with a higher $T_{\mathrm{m}}\left(41^{\circ} \mathrm{C}\right)$. As expected, the application of the DPPCcoated $\mathrm{Au}$ NS cores only provided the C-S NPs with irregularly shaped satellite NPs (Figure S3). The DPPC lipid bilayer is in its gelation state at room temperature, which could not serve as a capping ligand for the growing satellite NPs and regulate anisotropic growth. Following the growth of the satellites, the localized surface plasmon resonance (LSPR) wavelength of the core NPs showed a dramatic red shift corresponding to the increase in diameter and the plasmon coupling between the core and the satellites (Figure 1F). The spectra of the MGITC Raman reporter demonstrated a significantly higher SERS intensity of the C-S NPs compared to the cores with the same NP and MGITC concentrations, owing to the built-in electromagnetic hotspots within the $\mathrm{C}-\mathrm{S}$ NPs (Figure 1G).

Fluidity of Satellite NPs on the Core. Another observation was that there were round-shaped open pores on the satellite NP shell of each C-S NP (Figure 1C). A similar phenomenon also occurred in the $\mathrm{Au} \mathrm{NP}$-liposome hybrid NPs (Figure S2B). We deduced that these pores were generated during the drying process of the SEM sample preparation. The drying caused the wrinkling of the lipid bilayer on the core, and the movement of the satellites thus resulted in the formation of cracks in the densely packed 

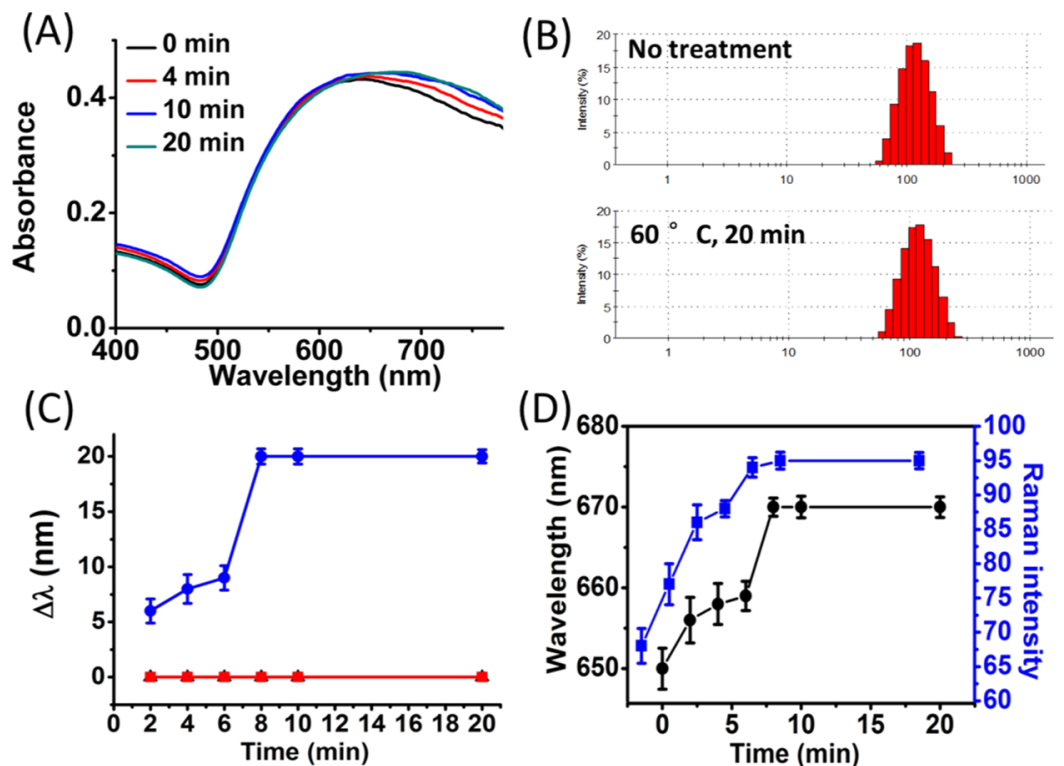

Figure 3. (A) Evolution of the UV-vis spectra of C-S NP solutions for $0,4,10$, and 20 min at $60{ }^{\circ} \mathrm{C}$. (B) DLS data of the C-S NP solutions with and without heating treatment. (C) Red-shift values of the LSPR peak of C-S NPs under different temperatures. (D) Variations in the SERS signal of the NT Raman reporter induced by the aggregation of the satellites, in accordance with the variation of the shift of the LSPR band of NPs. The data are presented as the mean value of three measurements with standard deviation.

nanorod array. To unveil the original morphology of the NPs in aqueous solution, we tried to stabilize the structure by further wrapping them with a polydopamine coating. As shown in Figure S4, the number and size of the pores dramatically decreased, and the spatial distribution of the satellites on the cores was more uniform.

These interesting pores observed via the SEM images provided a clue that the satellite NPs were movable on the core $\mathrm{NP}$, taking advantage of the fluidic property of the lipid bilayer. As shown in Figure 2A, the assembly of the nanorod satellite NPs into vertically orientated shell structures could be understood in terms of $(1)$ the electrostatic repulsion $\left(V_{\text {rep }}\right)$ between the adjacent nanorods provided by the net negative charge of the adsorbed lipid and AA, (2) the attractive van der Waals interactions $\left(V_{\mathrm{vdW}}\right)$ between the long hydrophobic chains of the lipid molecules on the nanorod side, (3) the attractive dipolar interaction potential $\left(V_{\text {dip }}\right)$ between the satellite NPs, ${ }^{56}$ and (4) the hydrophobic interactions between the lipid bilayers, which determined the fluidity of the lipid bilayer and the satellite NPs.

It has been reported that the free Au NPs in water are monodisperse because of the strong $V_{\text {rep }}$ between the capping ligands (such as citrate) and weak $V_{\text {dip }}$ as a result of the uniform capping layer. However, once the Au NPs adsorb onto a lipid membrane, the original capping layer becomes deformed, and the dipole moment is greatly enhanced. As a result, the attractive forces determined by the sum of $V_{\mathrm{vdW}}$ and $V_{\text {dip }}$ become greater than $V_{\text {rep. }}{ }^{29}$ Below $T_{\mathrm{m}}$, there was a tendency for the Au NPs to self-assemble, but it was prevented because the rigid gel phase of the lipid membrane locked the location of the NPs. Once the membrane was heated above the fusion temperature, the $\mathrm{Au} \mathrm{NP}$ mobility was increased and the NPs were able to self-assemble into aggregates.

Our C-S NP structure shares the similar phenomenon of heat-induced satellite NP aggregation, which could be confirmed by the SEM images and UV-vis spectra variations of the NPs before and after heating treatment. As shown in
Figure $2 \mathrm{~B}, \mathrm{C}$, after the heating treatment $\left(60^{\circ} \mathrm{C}\right)$, the original uniformly dispersed small nanorod satellites moved and aggregated in situ into larger, irregular "patches" on the $\mathrm{Au}$ core.

This phenomenon had never been reported for the $\mathrm{C}-\mathrm{S}$ NPs prepared by thiol group-containing molecules or protein as the linkers. In these $\mathrm{C}-\mathrm{S}$ NPs, the core and satellite NPs were linked by multiple binding groups of a single molecule through $\mathrm{Au}-\mathrm{S}$ and $\mathrm{Au}-\mathrm{N}$ bonds. The interaction was too strong for the satellites to move. By contrast, in our C-S NPs, the core and satellite NPs were adsorbed on the head of different lipid layers through strong $\mathrm{Au}-\mathrm{N}$ bonds and then linked through a relatively weak hydrophobic force between the alkyl long chains of the lipid bilayer. The increasing temperature can greatly decrease the hydrophobic force, providing a premise for the movement of the satellite NPs.

This structural change created a red shift in the UV-vis absorption peak of the C-S NPs, which could be dynamically monitored. The value reached as large as $20 \mathrm{~nm}$ within $10 \mathrm{~min}$ and then stabilized (Figure 3A). In addition, the surface plasmon resonance (SPR) peak slightly broadened because of the aggregation of the satellite NPs. The DLS data (Figure 3B) revealed that the monodispersity of the $\mathrm{C}-\mathrm{S}$ NPs was maintained before $[112.8 \mathrm{~nm}$, polydispersity index (PDI) $0.062]$ and after (112.7 nm, PDI 0.074) the treatment. The possibility of the disassembly of the C-S NPs or the aggregation among the $\mathrm{C}-\mathrm{S}$ NPs could also be precluded because almost no free satellite NPs could be detected in the SEM image and the DLS size distribution profile.

It is well-known that the lipid bilayer fluidity is greatly influenced by temperature. In our experiment, the aggregation of satellites was also temperature-dependent. As shown in the SEM images in Figure S5, no aggregation occurred at 25 and $40{ }^{\circ} \mathrm{C}$ after $3 \mathrm{~h}$, which was also revealed by the stable absorption peak in the UV-vis spectra of the C-S NPs (Figure 3C). However, with the temperature increasing to 60 ${ }^{\circ} \mathrm{C}$, the maximum absorption wavelength increased dramati- 

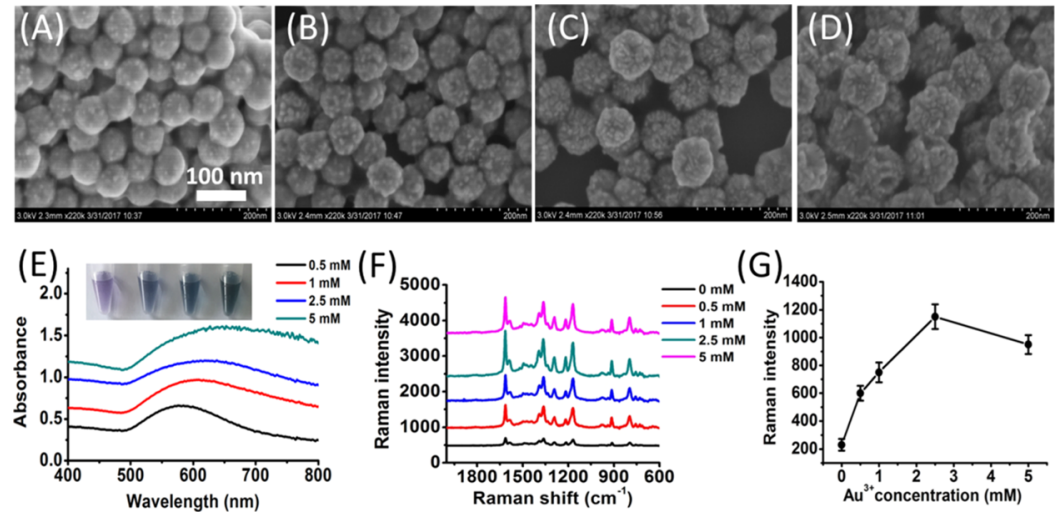

Figure 4. SEM images of C-S NPs with different morphologies synthesized using different concentrations of $\mathrm{Au}^{3+}:$ (A) 0.5 , (B) 1 , (C) 2.5 , and (D) $5 \mathrm{mM}$. Scale bar: $200 \mathrm{~nm}$. (E) Photographs of NP solutions prepared with $0.5,1,2.5$, and $5 \mathrm{mM} \mathrm{Au}^{3+}$ (from left to right), and the corresponding UV-vis absorption spectra. (F) SERS spectra of the MGITC Raman reporter from the NPs and (G) plots of SERS intensity as a function of the $\mathrm{Au}^{3+}$ concentration. Data are presented as the mean intensity of the $1164 \mathrm{~cm}^{-1}$ peak with standard deviation $(n=3)$.
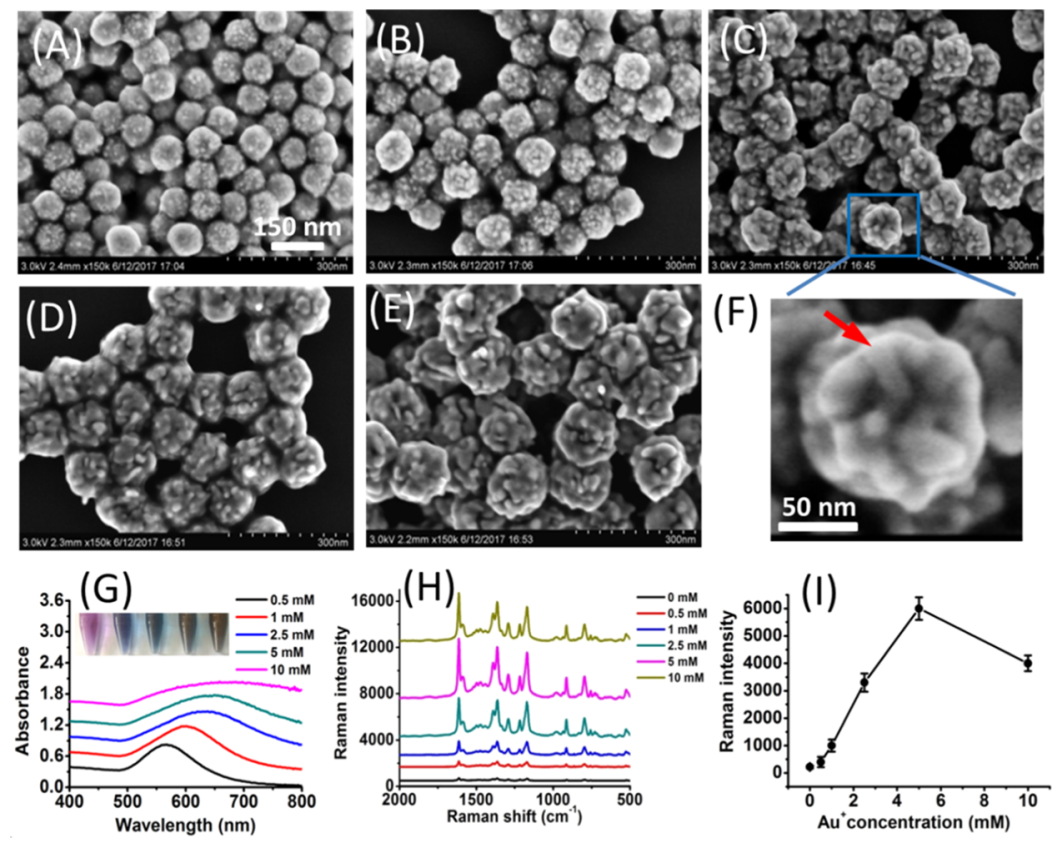

Figure 5. SEM images of C-S NPs with different morphologies synthesized using different concentrations of $\mathrm{Au}^{+}$: (A) 0.5 , (B) 1 , (C) 2.5, (D) 5, and (E) $10 \mathrm{mM}$. The concentration of AA was $0.1 \mathrm{M}$. (F) Enlarged image of (C) illustrating the gold nanorod satellite oriented vertically on the core. (G) Photographs of NP solutions prepared with $0.5,1,2.5,5$, and $10 \mathrm{mM} \mathrm{Au}^{+}$(from left to right), and the corresponding UV-vis absorption spectra. (H) SERS spectra of the MGITC Raman reporter from the NPs and (I) plots of SERS intensity as a function of Au concentration. The data are presented as the mean intensity of the $1164 \mathrm{~cm}^{-1}$ peak with standard deviation $(n=3)$.

cally within $10 \mathrm{~min}$. Simultaneously, the SERS signal of the Raman reporter NT adsorbed on the C-S NPs increased in a stepwise manner and then reached equilibrium, in accordance with the LSPR band of the red-shifted NPs. The heat-induced aggregation was irreversible, as were the changes in the SPR peak and SERS signal.

The above results also revealed that the adsorption of $\mathrm{Au}$ NPs affected the fluidity of the lipid bilayer. For the bare Lipoid E80 liposome, the lipid bilayer is fluidic at room temperature, which could be proved by the successful coating of the lipid bilayer on the Au NS core by just mixing the Au NS core and liposome at $25{ }^{\circ} \mathrm{C}$. By contrast, the satellite NP movement and optical feature change of the C-S NPs could be monitored only when the temperature increased to as high as $60{ }^{\circ} \mathrm{C}$. The greatly increased $T_{\mathrm{m}}$ indicated that the gold NP adsorption induced lipid gelation and inhibited the fluidity of the lipid bilayer.

Synthesis of $\mathrm{C}-\mathrm{S}$ NPs with $\mathrm{Au}^{3+}$. The effects of different synthesis conditions on the morphology and optical properties of the nanostructures were investigated in detail. First, different concentrations of chloroauric acid $\left(\mathrm{Au}^{3+}\right)$ were used as the reaction materials. The SEM images (Figure 4A-D) showed that when the concentration of $\mathrm{Au}^{3+}$ increased from 0.5 to 5.0 $\mathrm{mM}$, the number and size of the satellite NPs increased, and the gap between them became narrow. When the concentration of $\mathrm{Au}^{3+}$ was $0.5,12.5$, and $5 \mathrm{mM}$, the length of the satellite was approximately $5,11,25$, and $32 \mathrm{~nm}$, respectively (Figure S6). Figure 4E shows a series of colors of the NP product solutions that changed from purple, blue, green, and to dark green with an increasing concentration of $\mathrm{Au}^{3+}$. The corresponding $\mathrm{UV}$-vis absorption spectra indicated that the 

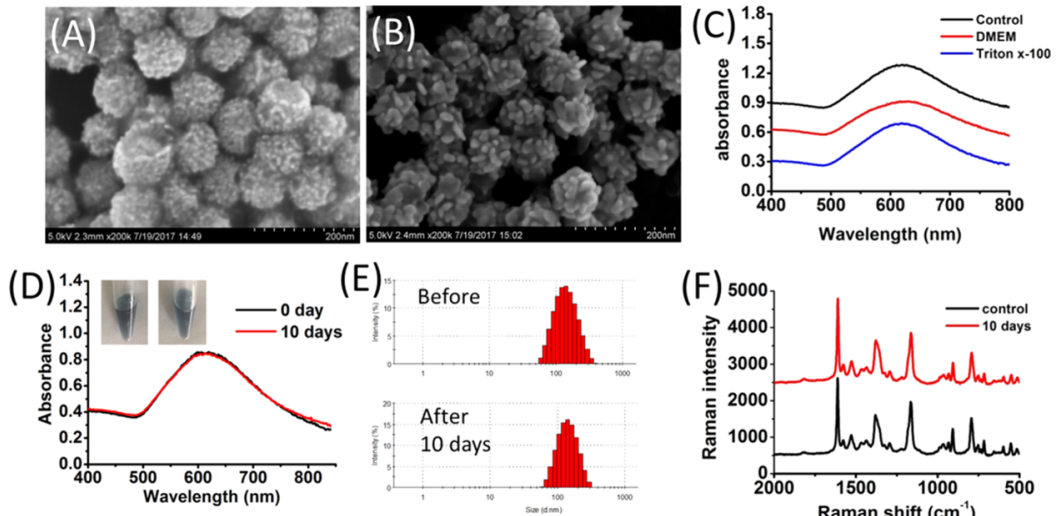

(E)
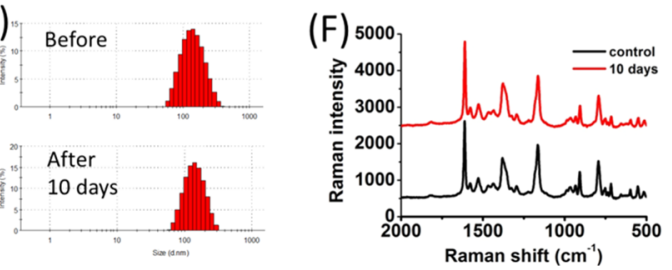

Figure 6. SEM images of $\mathrm{C}-\mathrm{S}$ NPs prepared with $\mathrm{Au}^{3+}(\mathrm{A})$ and $\mathrm{Au}^{+}$(B) after the treatment with $10 \%$ Triton X-100. (C) UV-vis absorption spectra of $\mathrm{C}-\mathrm{S}$ NPs prepared with $\mathrm{Au}^{+}$with the addition of DMEM and Triton X-100. No obvious changes could be observed compared to the control sample. (D) UV-vis absorption spectra of C-S NPs before and after 10 days of storage. The inset shows the solution before (left) and after (right) storage. (E) DLS size results of the NPs before $(131 \mathrm{~nm})$ and after $(127 \mathrm{~nm})$ storage. (F) SERS spectra of CV $\left(10^{-6} \mathrm{M}\right)$ obtained from the two sample solutions.

LSPR peaks broadened and red-shifted to $35,60,80$, and 110 $\mathrm{nm}$, respectively, compared with those of the spherical Au core. The SERS enhancement ability of each sample was investigated by using MGITC as a Raman reporter. As shown in Figure 4F, when the concentration of $\mathrm{Au}^{3+}$ increased from 0.5 to $2.5 \mathrm{mM}$, the SERS intensity of the sample increased continuously because of the formation of built-in hotspots. The maximum SERS intensity was approximately 6 times higher than the intensity of the Au core calculated from the Raman peak at $1164 \mathrm{~cm}^{-1}$ (Figure 4G). When more $\mathrm{Au}^{3+}$ was added $(5.0 \mathrm{mM})$, a decrease in SERS intensity was observed. This might be attributed to the fusion of gaps with excessive growth of the satellite NPs, which reduced the roughness of the C-S NPs and the number of hotspots. We further studied the generality of the synthesis protocol in terms of the Au core size. As shown in Figure S7, similar C-S NP structures could also be obtained when the size of the core NPs was as small as $23 \mathrm{~nm}$.

Effect of the AA Concentration. The effect of the AA concentration on morphology is illustrated in Figure S8A-C. With the increase in AA concentration from 0.01 to $1.0 \mathrm{M}$, the size of the satellite NPs decreased from $10 \mathrm{~nm}$ to approximately $5 \mathrm{~nm}$, whereas the coating density and morphological uniformity dramatically increased. The application of high concentrations of AA would accelerate the reduction reaction and produce more $A u$ seeds on the lipid bilayer surface at the initial stage, which would benefit the further growth of closely packed rod-like satellite shells. The LSPR band of the sample prepared with 1.0 M AA showed the largest red-shift value (Figure S8D), whereas the sample synthesized with $0.1 \mathrm{M}$ AA illustrated the best SERS enhancement ability because of the optimal satellite size and nanogap structures (Figure S8E).

Synthesis of $\mathrm{C}-\mathrm{S}$ NPs with $\mathrm{Au}^{+}$. Furthermore, we attempted to use $\mathrm{Au}^{+}$as the source of gold for the synthesis, and the SEM images are shown in Figure 5A-F. Similar waxberry-like $\mathrm{C}-\mathrm{S}$ NPs could also be obtained when the concentration of $\mathrm{Au}^{+}$was in the range of $2.5-5 \mathrm{mM}$ (Figure $5 C, D, G)$. However, it should be noted that the number of nanorod satellites on each core was diminished, and the average size (approximately $10 \mathrm{~nm}$ in width and $30 \mathrm{~nm}$ in length) was much larger than those synthesized from $\mathrm{Au}^{3+}$, which might be because fewer seeds were formed at the initial reaction stage. With a further increase in the $\mathrm{Au}^{+}$concentration to $10 \mathrm{mM}$, the nanorod array completely grew into an irregular solid shell with long cracks (Figure 5E). The color and LSPR spectra of the C-S NPs prepared from $\mathrm{Au}^{+}$showed a similar evolution in properties as the NPs synthesized from $\mathrm{Au}^{3+}$ (Figure 5G). The SERS signal variation also showed a "risefall" pattern, and the maximum intensity was reached when the sample was prepared with $5 \mathrm{mM}$ of $\mathrm{Au}^{+}$, which was nearly 30 times higher than that in the $\mathrm{Au}$ core (Figure $5 \mathrm{H}, \mathrm{I}$ ) and 5 times higher than the strongest value from the $\mathrm{Au}^{3+}$ samples, taking advantage of the better SERS enhancement ability of the larger satellite NPs. The slight signal decrease of the NPs prepared with $10 \mathrm{mM} \mathrm{Au}^{+}$was due to the loss of hotspots between the satellites and the C-S NPs after the overgrowth of the satellites. Considering the outstanding SERS enhancement ability, the $\mathrm{C}-\mathrm{S}$ NPs prepared by using $\mathrm{Au}^{+}$were used in the following SERS tag investigations.

Structural Stability of C-S NPs. Considering that the lipid bilayer is a fluidic "soft" coating and the structural integrity is not as rigid as the "solid" counterparts such as charged polymers and protein linkers, we paid special attention to the structural stability of the C-S NPs in our study. It is generally accepted that Triton X-100 dissolves the lipid bilayer and that either heating or the addition of cell culture medium can disrupt the integrity of the liposome. Therefore, we first tested how these sensitive factors of the phospholipid bilayer affected the morphology of our C-S NPs. As shown in Figure $6 \mathrm{~A}, \mathrm{~B}$, the addition of $10 \%$ Triton $\mathrm{X}-100$ to the $\mathrm{C}-\mathrm{S} \mathrm{NP}$ solution did not alter the morphologies of the NPs prepared with either $\mathrm{Au}^{3+}$ or $\mathrm{Au}^{+}$. The stability was also verified by monitoring the UV-vis absorption spectra after the addition of the DMEM medium (Figure 6C). Several works demonstrated that the stability of the liposomes with decorating $\mathrm{Au}$ clusters on the outer surface could be greatly enhanced, ${ }^{30,57}$ probably owing to lipid gelation at the adsorbed sites. In our C-S NPs, both the inner and outer surfaces of the lipid bilayer were adsorbed by the Au NPs; thus, the stability and structural integrity of the lipid bilayer might be further increased. Furthermore, after a 10-day storage of the NPs under ambient conditions, the LSPR property (Figure 6D), the DLS size (Figure 6E), as well as the SERS signal enhancement ability (Figure 6F) did not obviously change. 
Considering the practical biological applications of $\mathrm{C}-\mathrm{S}$ NPs as SERS tags, the possible effects of surface functionalization procedures on structural stability were tested. Antirabbit $\mathrm{IgG}$ was used as a model targeting ligand. After the complex $\mathrm{N}$ hydroxysuccinimide/ethyl(dimethylaminopropyl) carbodiimide reaction and purification procedures, the waxberry-like C-S NPs maintained their morphology without the detachment of the satellite NPs (Figure S9). The structural stability of the C-S NPs laid the foundation for further SERS tag preparation and bioimaging applications.

Raman Enhancement Ability of C-S NPs. The Raman enhancement ability of the $\mathrm{C}-\mathrm{S}$ NPs prepared from $\mathrm{Au}^{+}$was investigated by recording the SERS spectra of 11 Raman reporters to confirm the universal applicability of the NPs for the synthesis of SERS tags. As shown in Figure 7A, the characteristic Raman peaks could be observed for all reporters, with an optimal laser irradiation (632.8 or $780 \mathrm{~nm})$ determined by the maximum absorption wavelength of the reporters. We also compared the SERS intensities provided by our C-S NPs with those yielded by the spherical Au cores and well-known analogous counterparts, that is, $\mathrm{Au}$ nanoflowers fabricated by the reduction of chloroauric acid with HEPESbuffered solution ( $\mathrm{pH}$ 7.4; Figure S10). As shown in Figure 7B, among the three nanomaterials, our $\mathrm{C}-\mathrm{S}$ NPs provided the highest SERS intensities for all the tested Raman reporters, which were 2-6 times higher than those from the $\mathrm{Au}$ nanoflowers and 8-70 times higher than those from the spherical Au cores. The ideal SERS enhancement ability of the C-S NPs should be attributed to (1) the broad LSPR band covering the absorption peaks of all the reporters from the visible to the NIR region and the resultant Raman resonant effect that provided the tag with high sensitivity; (2) the entry of the Raman reporters into the hotspots via diffusion within the lipid bilayer to reach the maximum signal enhancement; ${ }^{58}$ and (3) more importantly, the numerous hotspots between the core and satellites, or between adjacent satellites, that provided a strong enhancement of the electromagnetic field. To prove this point, similar $\mathrm{C}-\mathrm{S}$ NPs were also prepared by using silica $\mathrm{NPs}$ as the cores (Figure S11). When the concentration of $\mathrm{Au}^{3+}$ added to the satellite NP preparation was adjusted to be the same $(2.5 \mathrm{mM})$, the absorbance peak positions of the two C-S NPs were similar, at $650 \mathrm{~nm}$. The Raman intensity of MGITC $\left(1620 \mathrm{~cm}^{-1}\right)$ was $390 \mathrm{cps}$ for the silica core-based C$S$ NPs and was only approximately one-third of that obtained from the Au NS core-based NPs (1010 cps).

Furthermore, the SERS enhancement factor of the C-S NPs prepared from $2.5 \mathrm{mM} \mathrm{Au}{ }^{+}$was calculated. Considering the potential application of the NPs as colloidal SERS tags, the analytical enhancement factor (AEF) of the NPs in colloidal dispersion was estimated by calculating the ratio between the SERS intensity for the selected mode of a given analyte $\left(I_{\text {SERS }}\right)$ and the corresponding nonenhanced Raman intensity $\left(I_{\mathrm{RS}}\right)$ under identical experimental conditions (laser wavelength and power, objectives, and exposure time), using the following equation

$$
\mathrm{AEF}=\frac{I_{\mathrm{SERS}} / C_{\mathrm{SERS}}}{I_{\mathrm{RS}} / C_{\mathrm{RS}}}
$$

where $C_{\mathrm{SERS}}$ and $C_{\mathrm{RS}}$ are the concentrations of the analyte in the SERS and Raman experiments, respectively. ${ }^{59,60}$ A small thiol-containing molecule, 4-nitrothiophenol (NT), was selected as the analyte because additional contribution to
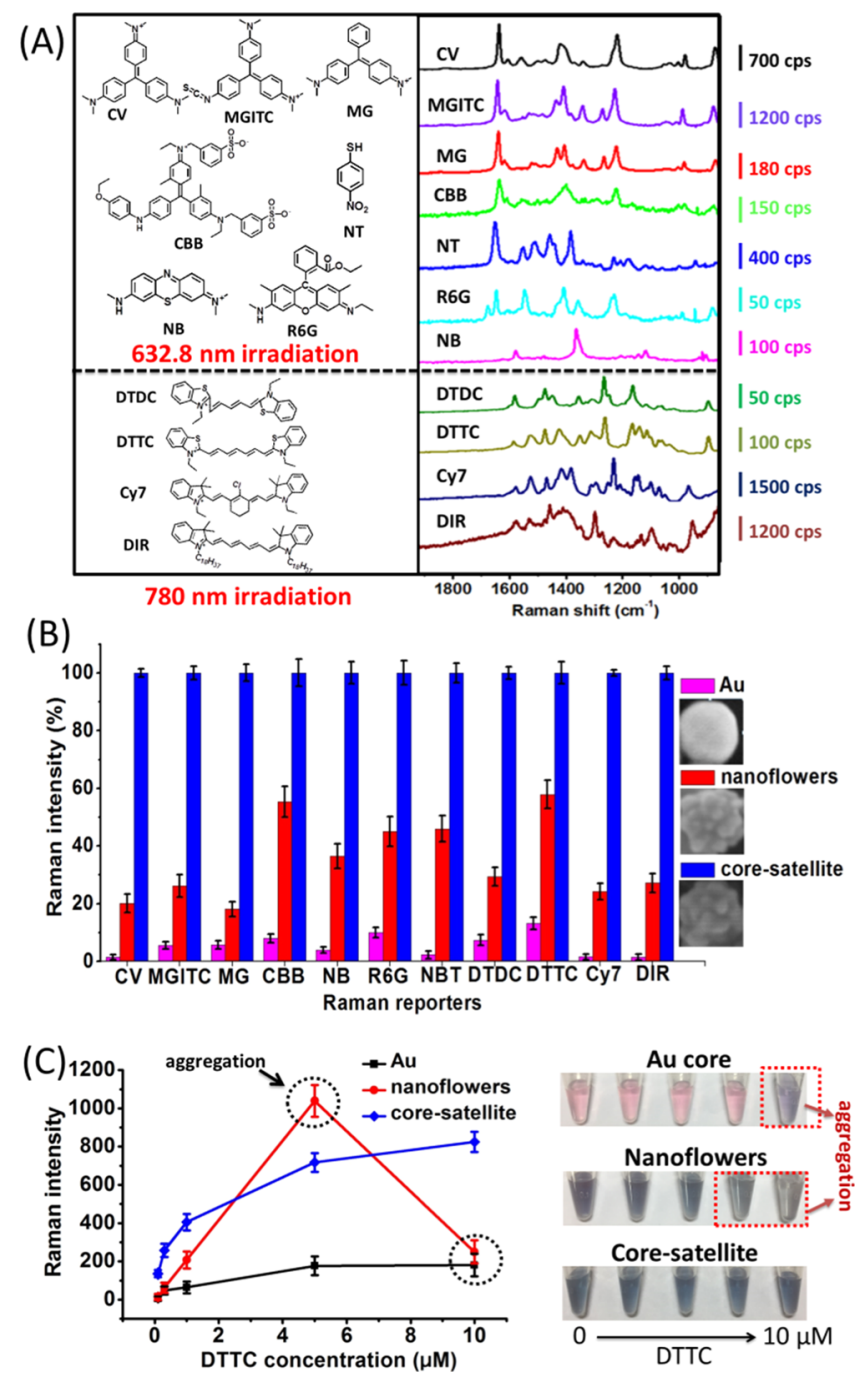

Figure 7. (A) SERS spectra of 11 representative Raman reporters from the C-S NP solutions. The concentration of NT was $3 \times 10^{-5}$ $\mathrm{M}$, and the concentration of other reporters was $3 \times 10^{-7} \mathrm{M}$. The signals of DTDC, DTTC, Cy7, and DIR were acquired by using 780 $\mathrm{nm}(4 \mathrm{~mW})$ irradiation, and the signals of the other reporters were acquired by using $632.8 \mathrm{~nm}(4 \mathrm{~mW})$ irradiation. (B) Comparison of the SERS enhancement ability of the Au core, Au nanoflower, and our $\mathrm{C}-\mathrm{S}$ NPs. The concentrations of the three NP solutions were adjusted to be the same according to the Au element $(0.25 \mathrm{mM})$. The concentration of NT was $3 \times 10^{-5} \mathrm{M}$, and the concentration of the other reporters was $3 \times 10^{-7} \mathrm{M}$. (C) SERS intensities of the three NP solutions prepared with DTTC at different concentrations (0.1, 0.3, 1, 5 , and $10 \mu \mathrm{M}$ ) (left panel), and the color of the three NP solutions corresponding to the dispersity of the NPs (right panel). Data are presented as the mean intensity of three measurements with standard deviation.

SERS originating from the molecular resonances upon $780 \mathrm{~nm}$ excitation could be avoided for the $\mathrm{AEF}$ estimation. With $C_{\mathrm{RS}}=$ $0.05 \mathrm{M}, C_{\mathrm{SERS}}=10^{-7} \mathrm{M}, I_{\mathrm{RS}}\left(1342 \mathrm{~cm}^{-1}\right)=110$, and $I_{\mathrm{SERS}}$ $\left(1342 \mathrm{~cm}^{-1}\right)=130$, the concentration of Au in the C-S NP solution was adjusted to $300 \mu \mathrm{M}$. The AEF was estimated to be $5.9 \times 10^{5}$, which was greater than the values of $5.6 \times 10^{4}$ and $1.2 \times 10^{5}$ presented in the literature for gold nanotriangle colloids ( 250 and $495 \mu \mathrm{M}$, respectively) with benzenethiol ${ }^{59}$ and 4-mercaptobenzoic acid ${ }^{60}$ as analytes. The AEFs of the $\mathrm{Au}$ core and $\mathrm{C}-\mathrm{S}$ NPs prepared from $2.5 \mathrm{mM}$ of $\mathrm{Au}^{3+}$ were also calculated to be $1.5 \times 10^{3}$ and $4.5 \times 10^{4}$, respectively. 
In addition to the superior SERS enhancement ability, the C-S NPs also exhibited the best colloidal stability during the SERS tag synthesis process. Different concentrations of the DTTC reporter (from 0.1 to $10 \mu \mathrm{M}$ ) were added to the three nanomaterial solutions. As shown in Figure 7C, the HEPEScapped $\mathrm{Au}$ nanoflower and the CTAC-stabilized Au core NPs aggregated when the concentration of the DTTC Raman reporter reached 5 and $10 \mu \mathrm{M}$, respectively, indicated by the color change of the solution. The aggregation of $\mathrm{Au}$ nanoflowers induced a sharp variation in the SERS signals. In contrast, the $\mathrm{C}-\mathrm{S} \mathrm{NPs}$ produced continuously growing signals with increasing concentrations of DTTC and maintained their monodispersity even when coincubated with the DTTC reporters at concentrations as high as $10 \mu \mathrm{M}$. Taking advantage of the colloidal stability, it was easy to fabricate monodisperse C-S NP-based SERS tags of high sensitivity (4 times higher than the DTTC-labeled nanoflowerbased tags in this case), without the need to finely adjust the concentration of the Raman reporters to prevent aggregation.

Cell Imaging. Good biocompatibility is the basis of cellular bioimaging. The cytotoxicity of the $\mathrm{C}-\mathrm{S}$ NPs was evaluated via the 3-(4,5-dimethylthiazol-2-yl)-2,5-diphenyltetrazolium bromide assay. As shown in Figure S12, cell viability was not significantly decreased at the highest dose of NPs $(100 \mu \mathrm{g}$ $\mathrm{mL}^{-1}$ ), but still remained at $90 \%$ at the NP concentration used for cellular imaging $(30 \mu \mathrm{g} / \mathrm{mL})$. The biocompatibility of the NPs was satisfactory for potential biological applications. Next, the in vitro imaging capability of the C-S NP-based SERS tags was demonstrated by employing the SMMC7721 cells as a model cell line. Dark-field imaging of the cells was employed to locate the cells, followed by Raman imaging (Figure 8). The
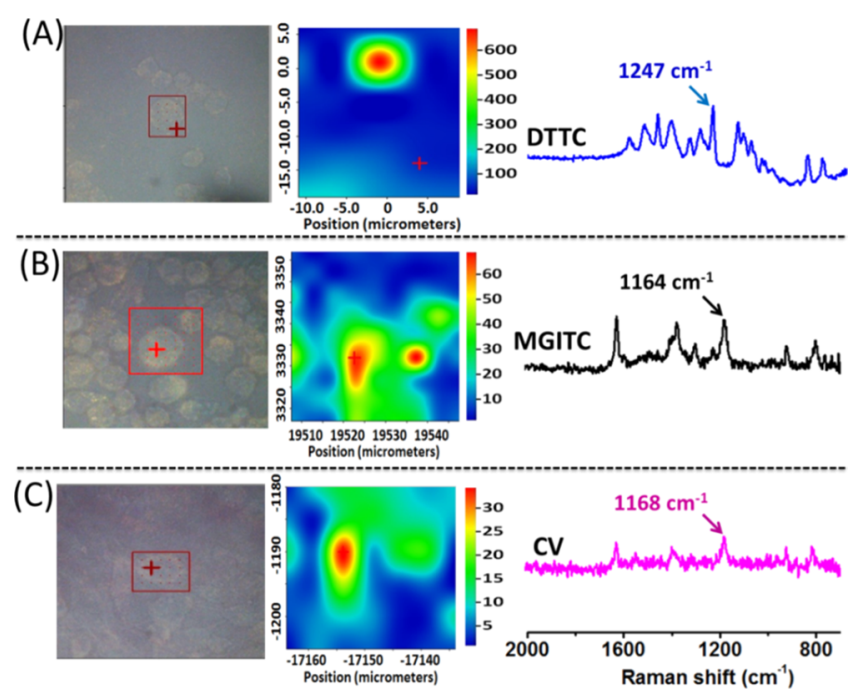

Figure 8. SERS imaging of the SMMC7721 cells using C-S NPbased SERS tags labeled with DTTC (A), MGITC (B), and CV (C) as the Raman reporters. The images were acquired after incubation with the tags for $3 \mathrm{~h}$. The right panel shows the spectra extracted from the intracellular regions.

dark-field images revealed the internalization of the tags. The Raman images of the cells were acquired using $632.8 \mathrm{~nm}$ (for MGITC- or CV-labeled tags) and $780 \mathrm{~nm}$ (for DTTC-labeled tags) laser irradiation. The intensity map of the characteristic Raman band of each reporter revealed the spatial distribution of the tags within the cells. The representative Raman spectra were also collected from the tag-labeled cells.
SERS Detection Investigation In Vivo. Finally, to demonstrate the potential applicability of the DTTC-labeled C-S NPs as NIR SERS tags for in vivo imaging, two proof-ofconcept experiments were performed in mouse models with a $780 \mathrm{~nm}$ laser irradiation. First, the deep tissue imaging capability of the tags was verified in a tumor-bearing nude mouse. The tag solution $(75 \mu \mathrm{g} / \mathrm{mL})$ was injected intratumorally at a depth of $6 \mathrm{~mm}$ (Figure 9A). As shown in Figure

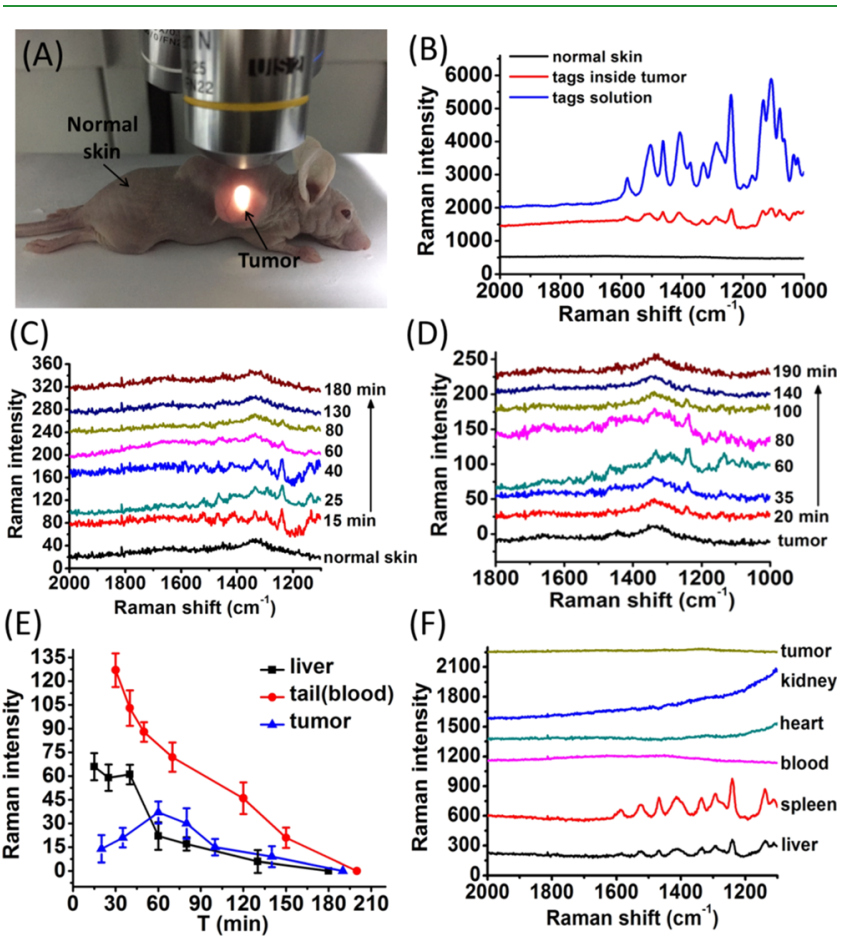

Figure 9. (A) In vivo SERS detection of a mouse injected intratumorally with DTTC-labeled NIR SERS tags by using a 780 $\mathrm{nm}$ laser irradiation. The type of the tumor was A549 human lung cancer cell xenograft, and the tumor size was approximately $15 \times 16 \times$ $6 \mathrm{~mm}$. (B) SERS spectra from the tags inside the tumor issue at a depth of $6 \mathrm{~mm}$, confirming the suitability of the SERS tag as an in vivo imaging agent. SERS spectra from the anatomical location of the liver (C) and tumor (D) of a living tumor-bearing mouse at various time points post injection through the tail vein. (E) Kinetics study of the SERS tag signal in liver, tail, and tumor sites. The data are presented as the mean intensity with standard deviation $(n=3)$. (F) Ex vivo SERS spectra obtained from the tumor and the main organs of the same mouse after sacrifice ( $3.5 \mathrm{~h}$ post injection) and the stripping off the overlying skin.

9B, the injected region exhibited a SERS signal that wellmatched with the SERS spectrum of the tag, illustrating that the ultrasensitivity of the tag would enable deep tissue imaging. In the second experiment, we injected the tags into a mouse through the tail vein and monitored the in vivo SERS signals at the sites of the liver and the tail. The spectra from the liver at different time intervals are illustrated in Figure 9C. The obvious tag signals from the liver could penetrate the skin and be monitored, and the maximum intensity could be recorded within $15 \mathrm{~min}$, followed by a decrease with prolonged time. Similar results were also obtained from the tail. Figure 9D showed that the intensity at the tumor site slowly increased and reached the maximum in $1 \mathrm{~h}$, and then the signal vanished after $2 \mathrm{~h}$. Figure 9E showed the temporal evolution of the intensity at the three sites. The signal from the liver was nearly 
stable for the first $40 \mathrm{~min}$ and then gradually decreased. This signal-stable period revealed that the tags were taken up by the Kupffer cells of the reticuloendothelial system and, as a result, were naturally accumulated in the liver. ${ }^{61}$ In contrast, the signal detected at the tail site underwent a continuous decrease because of the rapid clearance of the tags from the blood. The increasing and decreasing signal at the tumor indicated the slow passive uptake and clearance of the SERS tags. After $3.5 \mathrm{~h}$, the mouse was executed, and the SERS signals of the tumor and main organs were measured after stripping off the overlying skin. The liver and spleen demonstrated strong signals, whereas almost no signals could be detected from the heart, kidney, or blood, and tumor (Figure 9F), which provided a preliminary evidence that the NPs had varied distribution and accumulation behaviors in these locations.

\section{CONCLUSIONS}

In summary, we developed novel C-S NPs by using the fluidic lipid bilayer as a cross-linker for the first time. An interesting waxberry-like structure was obtained, and the satellites on the cores were found to be movable under heating conditions. The C-S NPs exhibited satisfactory SERS enhancement ability for various kinds of Raman reporters, which was attributed to the three-dimensional distribution of the SERS hotspots between the Au core and its satellites, separated by a gap formed by the Raman reporter-accessible lipid bilayers. Moreover, the capability of C-S NP-based SERS tags, both in the visible and the NIR optical window for bioimaging, was demonstrated through a proof-of-concept of live-cell and in vivo imaging applications. The SERS tags have promising applications for in vivo bioanalysis and preclinical diagnosis.

\section{ASSOCIATED CONTENT}

\section{S Supporting Information}

The Supporting Information is available free of charge on the ACS Publications website at DOI: 10.1021/acsami.8b06253.

Zeta potential and DLS data of the C-S NPs; TEM image of lipid bilayer-coated Au core; FTIR spectra of lipoid E80, lipid-coated Au NS core, and CTAC-Au NS core; SEM images of liposome-Au hybrids and C-S NPs prepared by using DPPC lipid bilayer-coated $\mathrm{Au}$ spheres as cores; $\mathrm{C}-\mathrm{S}$ NPs coated with polydopamine polymer; SEM images of C-S NPs after heat treatment, prepared with $23 \mathrm{~nm} \mathrm{Au}$ cores, and with different concentrations of AA; SEM images of goat antirabbit IgG-functionalized C-S NP-based SERS tags; SEM image of nanoflowers and $\mathrm{C}-\mathrm{S}$ NPs prepared with 1.5 $\mathrm{mM} \mathrm{Au}{ }^{+}$and the corresponding UV-vis absorption spectra; SEM images of silica core-based C-S NPs and the optical properties; and cell viability of $\mathrm{C}-\mathrm{S}$ NPs (PDF)

\section{AUTHOR INFORMATION}

\section{Corresponding Authors}

*E-mail: yqwang@yic.ac.cn (Y.W.).

*E-mail: lxchen@yic.ac.cn. Tel: +86 535 2109130. Fax: +86 5352109130 (L.C.).

\section{ORCID}

Lingxin Chen: 0000-0002-3764-3515

\section{Notes}

The authors declare no competing financial interest.

\section{ACKNOWLEDGMENTS}

Financial support from the National Natural Science Foundation of China (81573393 and 21575159), the Science and Technology Development Plan of Shandong Province of China (2014GGX109001), the Youth Innovation Promotion Association CAS (2017256), the Defense Science and Technology Innovation Fund of the Chinese Academy of Sciences (CXJJ-17-Q148), and the Instrument Developing Project of the Chinese Academy of Sciences (YZ201662) is gratefully acknowledged.

\section{REFERENCES}

(1) Wang, Z.; Zong, S.; Wu, L.; Zhu, D.; Cui, Y. SERS-Activated Platforms for Immunoassay: Probes, Encoding Methods, and Applications. Chem. Rev. 2017, 117, 7910-7963.

(2) Cialla-May, D.; Zheng, X.-S.; Weber, K.; Popp, J. Recent Progress in Surface-Enhanced Raman Spectroscopy for Biological and Biomedical Applications: From Cells to Clinics. Chem. Soc. Rev. 2017, 46, 3945-3961.

(3) Reguera, J.; Langer, J.; Jiménez de Aberasturi, D.; Liz-Marzán, L. M. Anisotropic Metal Nanoparticles for Surface Enhanced Raman Scattering. Chem. Soc. Rev. 2017, 46, 3866-3885.

(4) Laing, S.; Jamieson, L. E.; Faulds, K.; Graham, D. SurfaceEnhanced Raman Spectroscopy for in Vivo Biosensing. Nat. Rev. Chem. 2017, 1, 0060.

(5) Qiu, L.; Wang, W.; Zhang, A.; Zhang, N.; Lemma, T.; Ge, H.; Toppari, J. J.; Hytönen, V. P.; Wang, J. Core-Shell Nanorod Columnar Array Combined with Gold Nanoplate-Nanosphere Assemblies Enable Powerful in Situ SERS Detection of Bacteria. ACS Appl. Mater. Interfaces 2016, 8, 24394-24403.

(6) Lin, D.; Qin, T.; Wang, Y.; Sun, X.; Chen, L. Graphene Oxide Wrapped SERS Tags: Multifunctional Platforms toward Optical Labeling, Photothermal Ablation of Bacteria, and the Monitoring of Killing Effect. ACS Appl. Mater. Interfaces 2014, 6, 1320-1329.

(7) Lin, M.; Wang, Y.; Sun, X.; Wang, W.; Chen, L. Elastic Property of Mesoporous Silica Shell: For Dynamic Surface Enhanced Raman Scattering Ability Monitoring of Growing Noble Metal Nanostructures via a Simplified Spatially Confined Growth Method. ACS Appl. Mater. Interfaces 2015, 7, 7516-7525.

(8) Su, X.; Wang, Y.; Wang, W.; Sun, K.; Chen, L. Phospholipid Encapsulated Aunr@Ag/Au Nanosphere SERS Tags with Environmental Stimulus Responsive Signal Property. ACS Appl. Mater. Interfaces 2016, 8, 10201-10211.

(9) Wang, Y.; Yan, B.; Chen, L. SERS Tags: Novel Optical Nanoprobes for Bioanalysis. Chem. Rev. 2013, 113, 1391-1428.

(10) Huang, Z.; Meng, G.; Huang, Q.; Chen, B.; Lu, Y.; Wang, Z.; Zhu, X.; Sun, K. Surface-Enhanced Raman Scattering from Plasmonic Ag-Nanocube@Au-Nanospheres Core@Satellites. J. Raman Spectrosc. 2017, 48, 217-223.

(11) Dai, L.; Song, L.; Huang, Y.; Zhang, L.; Lu, X.; Zhang, J.; Chen, T. Bimetallic Au/Ag Core-Shell Superstructures with Tunable Surface Plasmon Resonance in the near-Infrared Region and High Performance Surface-Enhanced Raman Scattering. Langmuir 2017, 33, 53785384.

(12) Schütz, M.; Schlücker, S. Molecularly Linked 3d Plasmonic Nanoparticle Core/Satellite Assemblies: SERS Nanotags with SingleParticle Raman Sensitivity. Phys. Chem. Chem. Phys. 2015, 17, 2435624360.

(13) Gellner, M.; Steinigeweg, D.; Ichilmann, S.; Salehi, M.; Schütz, M.; Kömpe, K.; Haase, M.; Schlücker, S. 3d Self-Assembled Plasmonic Superstructures of Gold Nanospheres: Synthesis and Characterization at the Single-Particle Level. Small 2011, 7, 34453451.

(14) Maiti, K. K.; Dinish, U. S.; Samanta, A.; Vendrell, M.; Soh, K.S.; Park, S.-J.; Olivo, M.; Chang, Y.-T. Multiplex Targeted in Vivo Cancer Detection Using Sensitive near-Infrared SERS Nanotags. Nano Today 2012, 7, 85-93. 
(15) Chang, H.; Kang, H.; Yang, J.-K.; Jo, A.; Lee, H.-Y.; Lee, Y.-S.; Jeong, D. H. Ag Shell-Au Satellite Hetero-Nanostructure for UltraSensitive, Reproducible, and Homogeneous NIR SERS Activity. ACS Appl. Mater. Interfaces 2014, 6, 11859-11863.

(16) Xie, W.; Walkenfort, B.; Schlücker, S. Label-Free SERS Monitoring of Chemical Reactions Catalyzed by Small Gold Nanoparticles Using 3d Plasmonic Superstructures. J. Am. Chem. Soc. 2013, 135, 1657-1660.

(17) Gandra, N.; Abbas, A.; Tian, L.; Singamaneni, S. Plasmonic Planet-Satellite Analogues: Hierarchical Self-Assembly of Gold Nanostructures. Nano Lett. 2012, 12, 2645-2651.

(18) Gandra, N.; Portz, C.; Nergiz, S. Z.; Fales, A.; Vo-Dinh, T.; Singamaneni, S. Inherently Stealthy and Highly Tumor-Selective Gold Nanoraspberries for Photothermal Cancer Therapy. Sci. Rep. 2015, 5, 10311.

(19) Shiohara, A.; Novikov, S. M.; Solís, D. M.; Taboada, J. M.; Obelleiro, F.; Liz-Marzán, L. M. Plasmon Modes and Hot Spots in Gold Nanostar-Satellite Clusters. J. Phys. Chem. C 2015, 119, 1083610843.

(20) Indrasekara, A. S. D. S.; Thomas, R.; Fabris, L. Plasmonic Properties of Regiospecific Core-Satellite Assemblies of Gold Nanostars and Nanospheres. Phys. Chem. Chem. Phys. 2015, 17, 21133-21142.

(21) Dey, P.; Zhu, S.; Thurecht, K. J.; Fredericks, P. M.; Blakey, I. Self assembly of plasmonic core-satellite nano-assemblies mediated by hyperbranched polymer linkers. J. Mater. Chem. B 2014, 2, 28272837.

(22) Pal, S.; Sharma, J.; Yan, H.; Liu, Y. Stable Silver NanoparticleDNA Conjugates for Directed Self-Assembly of Core-Satellite SilverGold Nanoclusters. Chem. Commun. 2009, 6059-6061.

(23) Zhu, Y.; Jiang, X.; Wang, H.; Wang, S.; Wang, H.; Sun, B.; Su, Y.; He, Y. A Poly Adenine-Mediated Assembly Strategy for Designing Surface-Enhanced Resonance Raman Scattering Substrates in Controllable Manners. Anal. Chem. 2015, 87, 6631-6638.

(24) Höller, R. P. M.; Dulle, M.; Thomä, S.; Mayer, M.; Steiner, A. M.; Förster, S.; Fery, A.; Kuttner, C.; Chanana, M. Protein-Assisted Assembly of Modular 3d Plasmonic Raspberry-Like Core/Satellite Nanoclusters: Correlation of Structure and Optical Properties. ACS Nano 2016, 10, 5740-5750.

(25) Kumar, S.; Kumar, A.; Kim, G.-H.; Rhim, W.-K.; Hartman, K. L.; Nam, J.-M. Myoglobin and Polydopamine-Engineered Raman Nanoprobes for Detecting, Imaging, and Monitoring Reactive Oxygen Species in Biological Samples and Living Cells. Small 2017, 13, 1701584.

(26) Lee, J.-H.; Oh, J.-W.; Nam, S. H.; Cha, Y. S.; Kim, G.-H.; Rhim, W.-K.; Kim, N. H.; Kim, J.; Han, S. W.; Suh, Y. D.; Nam, J.-M. Synthesis, Optical Properties, and Multiplexed Raman Bio-Imaging of Surface Roughness-Controlled Nanobridged Nanogap Particles. Small 2016, 12, 4726-4734.

(27) Tian, L.; Fei, M.; Tadepalli, S.; Morrissey, J. J.; Kharasch, E. D.; Singamaneni, S. Bio-Enabled Gold Superstructures with Built-in and Accessible Electromagnetic Hotspots. Adv. Healthcare Mater. 2015, 4, $1502-1509$.

(28) Tian, L.; Tadepalli, S.; Fei, M.; Morrissey, J. J.; Kharasch, E. D.; Singamaneni, S. Off-Resonant Gold Superstructures as Ultrabright Minimally Invasive Surface-Enhanced Raman Scattering (SERS) Probes. Chem. Mater. 2015, 27, 5678-5684.

(29) Sugikawa, K.; Kadota, T.; Yasuhara, K.; Ikeda, A. Anisotropic Self-Assembly of Citrate-Coated Gold Nanoparticles on Fluidic Liposomes. Angew. Chem., Int. Ed. 2016, 55, 4059-4063.

(30) Zhang, L.; Granick, S. How to Stabilize Phospholipid Liposomes (Using Nanoparticles). Nano Lett. 2006, 6, 694-698.

(31) Alipour, E.; Halverson, D.; McWhirter, S.; Walker, G. C. Phospholipid Bilayers: Stability and Encapsulation of Nanoparticles. Annu. Rev. Phys. Chem. 2017, 68, 261-283.

(32) Orendorff, C. J.; Alam, T. M.; Sasaki, D. Y.; Bunker, B. C.; Voigt, J. A. Phospholipid-Gold Nanorod Composites. ACS Nano 2009, 3, 971-983.
(33) Stewart, A. F.; Lee, A.; Ahmed, A.; Ip, S.; Kumacheva, E.; Walker, G. C. Rational Design for the Controlled Aggregation of Gold Nanorods Via Phospholipid Encapsulation for Enhanced Raman Scattering. ACS Nano 2014, 8, 5462-5467.

(34) Tam, N. C. M.; McVeigh, P. Z.; MacDonald, T. D.; Farhadi, A.; Wilson, B. C.; Zheng, G. Porphyrin-Lipid Stabilized Gold Nanoparticles for Surface Enhanced Raman Scattering Based Imaging. Bioconjugate Chem. 2012, 23, 1726-1730.

(35) Tam, N. C. M.; Scott, B. M. T.; Voicu, D.; Wilson, B. C.; Zheng, G. Facile Synthesis of Raman Active Phospholipid Gold Nanoparticles. Bioconjugate Chem. 2010, 21, 2178-2182.

(36) Ip, S.; MacLaughlin, C. M.; Gunari, N.; Walker, G. C. Phospholipid Membrane Encapsulation of Nanoparticles for SurfaceEnhanced Raman Scattering. Langmuir 2011, 27, 7024-7033.

(37) Matthews, J. R.; Payne, C. M.; Hafner, J. H. Analysis of Phospholipid Bilayers on Gold Nanorods by Plasmon Resonance Sensing and Surface-Enhanced Raman Scattering. Langmuir 2015, 31 9893-9900.

(38) Wright, A. J.; Richens, J. L.; Bramble, J. P.; Cathcart, N.; Kitaev, V.; O'Shea, P.; Hudson, A. J. Surface-Enhanced Raman Scattering Measurement from a Lipid Bilayer Encapsulating a Single Decahedral Nanoparticle Mediated by an Optical Trap. Nanoscale 2016, 8, 16395-16404.

(39) Suga, K.; Yoshida, T.; Ishii, H.; Okamoto, Y.; Nagao, D.; Konno, M.; Umakoshi, H. Membrane Surface-Enhanced Raman Spectroscopy for Sensitive Detection of Molecular Behavior of Lipid Assemblies. Anal. Chem. 2015, 87, 4772-4780.

(40) Troutman, T. S.; Barton, J. K.; Romanowski, M. Biodegradable Plasmon Resonant Nanoshells. Adv. Mater. 2008, 20, 2604-2608.

(41) Leung, S. J.; Kachur, X. M.; Bobnick, M. C.; Romanowski, M. Wavelength-Selective Light-Induced Release from Plasmon Resonant Liposomes. Adv. Funct. Mater. 2011, 21, 1113-1121.

(42) Leung, S. J.; Romanowski, M. Molecular Catch and Release: Controlled Delivery Using Optical Trapping with Light-Responsive Liposomes. Adv. Mater. 2012, 24, 6380-6383.

(43) Troutman, T. S.; Leung, S. J.; Romanowski, M. Light-Induced Content Release from Plasmon-Resonant Liposomes. Adv. Mater. 2009, 21, 2334-2338.

(44) Kwon, H. J.; Byeon, Y.; Jeon, H. N.; Cho, S. H.; Han, H. D.; Shin, B. C. Gold Cluster-Labeled Thermosensitive Liposmes Enhance Triggered Drug Release in the Tumor Microenvironment by a Photothermal Effect. J. Controlled Release 2015, 216, 132-139.

(45) Rengan, A. K.; Bukhari, A. B.; Pradhan, A.; Malhotra, R.; Banerjee, R.; Srivastava, R.; De, A. In Vivo Analysis of Biodegradable Liposome Gold Nanoparticles as Efficient Agents for Photothermal Therapy of Cancer. Nano Lett. 2015, 15, 842-848.

(46) Rengan, A. K.; Jagtap, M.; De, A.; Banerjee, R.; Srivastava, R. Multifunctional Gold Coated Thermo-Sensitive Liposomes for Multimodal Imaging and Photo-Thermal Therapy of Breast Cancer Cells. Nanoscale 2014, 6, 916-923.

(47) Lum, W.; Bruzas, I.; Gorunmez, Z.; Unser, S.; Beck, T.; Sagle, L. Novel Liposome-Based Surface-Enhanced Raman Spectroscopy (SERS) Substrate. J. Phys. Chem. Lett. 2017, 8, 2639-2646.

(48) Zheng, Y.; Zhong, X.; Li, Z.; Xia, Y. Successive, Seed-Mediated Growth for the Synthesis of Single-Crystal Gold Nanospheres with Uniform Diameters Controlled in the Range of 5-150 nm. Part. Part. Syst. Charact. 2014, 31, 266-273.

(49) Bai, T.; Tan, Y.; Zou, J.; Nie, M.; Guo, Z.; Lu, X.; Gu, N. AuBr2-Engaged Galvanic Replacement for Citrate-Capped Au-Ag Alloy Nanostructures and Their Solution-Based Surface-Enhanced Raman Scattering Activity. J. Phys. Chem. C 2015, 119, 28597-28604.

(50) Sun, C.; Zhang, L.; Zhang, R.; Gao, M.; Zhang, X. Facilely Synthesized Polydopamine Encapsulated Surface-Enhanced Raman Scattering (SERS) Probes for Multiplex Tumor Associated Cell Surface Antigen Detection Using SERS Imaging. RSC Adv. 2015, 5 , 72369-72372.

(51) Xie, J.; Zhang, Q.; Lee, J. Y.; Wang, D. I. C. The Synthesis of SERS-Active Gold Nanoflower Tags for in Vivo Applications. ACS Nano 2008, 2, 2473-2480. 
(52) Pawlikowska-Pawlega, B.; Misiak, L. E.; Zarzyka, B.; Paduch, R.; Gawron, A.; Gruszecki, W. I. Localization and Interaction of Genistein with Model Membranes Formed with Dipalmitoylphosphatidylcholine (DPPC). Biochim. Biophys. Acta, Biomembr. 2012, 1818, 1785-1793.

(53) Feng, Y.; Wang, Y.; He, J.; Song, X.; Tay, Y. Y.; Hng, H. H.; Ling, X. Y.; Chen, H. Achieving Site-Specificity in Multistep Colloidal Synthesis. J. Am. Chem. Soc. 2015, 137, 7624-7627.

(54) Jia, W.; Li, J.; Jiang, L. Synthesis of Highly Branched Gold Nanodendrites with a Narrow Size Distribution and Tunable NIR and SERS Using a Multiamine Surfactant. ACS Appl. Mater. Interfaces 2013, 5, 6886-6892.

(55) Vijayaraghavan, P.; Liu, C.-H.; Hwang, K. C. Synthesis of Multibranched Gold Nanoechinus Using a Gemini Cationic Surfactant and Its Application for Surface Enhanced Raman Scattering. ACS Appl. Mater. Interfaces 2016, 8, 23909-23919.

(56) Xi, C.; Marina, P. F.; Xia, H.; Wang, D. Directed Self-Assembly of Gold Nanoparticles into Plasmonic Chains. Soft Matter 2015, 11, $4562-4571$.

(57) Liu, J. Interfacing Zwitterionic Liposomes with Inorganic Nanomaterials: Surface Forces, Membrane Integrity, and Applications. Langmuir 2016, 32, 4393-4404.

(58) Kühler, P.; Weber, M.; Lohmüller, T. Plasmonic Nanoantenna Arrays for Surface-Enhanced Raman Spectroscopy of Lipid Molecules Embedded in a Bilayer Membrane. ACS Appl. Mater. Interfaces 2014, 6, 8947-8952.

(59) Scarabelli, L.; Coronado-Puchau, M.; Giner-Casares, J. J.; Langer, J.; Liz-Marzán, L. M. Monodisperse Gold Nanotriangles: Size Control, Large-Scale Self-Assembly, and Performance in SurfaceEnhanced Raman Scattering. ACS Nano 2014, 8, 5833-5842.

(60) Kuttner, C.; Mayer, M.; Dulle, M.; Moscoso, A.; LópezRomero, J. M.; Förster, S.; Fery, A.; Pérez-Juste, J.; Contreras-Cáceres, R. Seeded Growth Synthesis of Gold Nanotriangles: Size Control, Saxs Analysis, and SERS Performance. ACS Appl. Mater. Interfaces 2018, 10, 11152-11163.

(61) Zavaleta, C. L.; Smith, B. R.; Walton, I.; Doering, W.; Davis, G.; Shojaei, B.; Natan, M. J.; Gambhir, S. S. Multiplexed Imaging of Surface Enhanced Raman Scattering Nanotags in Living Mice Using Noninvasive Raman Spectroscopy. Proc. Natl. Acad. Sci. U.S.A. 2009, 106, 13511-13516. 